\title{
Neural Network Based Classification of Myocardial Infarction: A Comparative Study of Wavelet and Fourier Transforms
}

\author{
Fawzi Al-Naima ${ }^{1} \&$ Ali Al-Timemy ${ }^{2}$ \\ ${ }^{1}$ Computer Engineering Department, College of Engineering, \\ Nahrain University, Baghdad, \\ Iraq \\ ${ }^{2}$ School of Computing, Communications and Electronics, \\ University of Plymouth, Plymouth, Devon, \\ United Kingdom
}

\section{Introduction}

The development of automated systems for medical diagnosis is a significant challenge faced by physicians, engineers and computer scientists. Such system requires a data set sufficiently large in order to be considered a reliable statistical sample (Karuiannis \& Venetsanepouious, 1997). Conventional methods of monitoring and diagnosing cardiac abnormalities rely on detecting the presence of particular signal features by human observer. Due to the large number of patients in intensive care units and the need for continuous observation of such conditions; several techniques for automated abnormality detection have been developed in recent years to attempt to solve this problem. Such techniques work by transforming the mostly qualitative diagnostic criteria into a more objective quantitative signal feature classification problem (Owis et al., 2002).

Computer technology has an important role in structuring biological systems. The explosive growth of high-performance computing techniques in recent years with regard to the development of good and accurate models of biological systems has contributed significantly to new approaches to fundamental problems of modelling transient behaviour of biological systems. Computer based analytical tools for the in-depth study and classification of data over day-long intervals can be very useful in diagnostics (Acharya et al., 2004).

The ECG signal represents the changes in electrical potential during the cardiac cycle as recorded between surface electrodes on the human body. The characteristic shape of this signal is the result of an action potential that propagates within the heart and causes the contraction of the various portions of cardiac muscle. This internal excitation starts at the sinus node which acts as a pacemaker, and then spreads to the atria, this generates the characteristic P wave in the ECG. The cardiac excitation then reaches the ventricles giving rise to the characteristic QRS complex. After that the ventricles repolarises corresponding to 
the T wave of the ECG. The automatic detection and timing of these waves is important for diagnostic purposes (Unser \& Aldroubi, 1996; Prasad \& Sahambi, 2003).

The classification of ECG signals into different disease categories is a complex pattern recognition task. In conventional systems, a typical heart beat is identified from the ECG. The $\mathrm{P}, \mathrm{QRS}$ and $\mathrm{T}$ waves are characterized using measurements such as magnitude, duration and area. Classification is performed on these measurements, but the measurements of $\mathrm{P}, \mathrm{QRS}$ and T sections vary significantly even among normal beats and can lead to misclassification (Kim et al., 2001).

Electrocardiograms are signals that originate from the action of the human heart. The ECG is the graphical representation of the potential difference between two points on body surface, versus time. Its historical development has resulted in a tool for clinical diagnosis, the 12lead ECG.

ECG signals are largely employed as a diagnostic tool in clinical practice in order to assess the cardiac status of a subject. They are used to examine ambulatory patients who are at rest during a recording or performing an exercise program and also patients in intensive care units. ECG recordings are examined by a physician who visually checks features of the signal and estimates the most important parameters of the signal. Using his expertise the physician judges the status of a patient. The recognition and the analysis of the ECG signal is difficult, since their size and form may change eventually and there can be a considerable amount of noise in the signal. Since the processing of ECG signal is a very important step in the process of ECG examination by physicians many tools, methods and algorithms from signal processing theory have been proposed, described and implemented. The Wavelet Transform (WT) is a new and promising set of tools and techniques for doing this. Wavelets have generated a tremendous interest in both theoretical and applied areas, especially over the past few years. A number of papers, already large, continues to grow, thus progress is being made at a rapid pace (Anan et al. 1995; Novak, 2000; Afsar \& Arif, 2008).

\section{Wavelets Applications in Medicine}

The results of the studies in the literature have demonstrated that the WT is the most promising method to extract features from the ECG signals. The WT can be thought of as an extension of the classic Fourier transform, except that, instead of working on a single scale (time or frequency), it works on a multi-scale basis. This multi-scale feature of the WT allows the decomposition of a signal into a number of scales, each scale representing a particular coarseness of the signal under study. The WT provides very general techniques, which can be applied to many tasks in signal processing. One very important application is the ability to compute and manipulate data in compressed parameters which are often called features. Thus, the ECG signal, consisting of many data points, can be compressed into a few parameters. These parameters characterize the behavior of the ECG signal. These features of using a smaller number of parameters to represent the ECG signal are particularly important for recognition and diagnostic purposes (Güler \& Übeyli, 2004).

The WT of a signal consists of breaking up a signal into shifted and scaled versions of a reference (mother) wavelet and has good properties of time and frequency localization. It is robust to time varying signal analysis. The wavelet coefficients represent measures of similarity of the local shape of the signal to the mother wavelet under different shifts and scales (Unser \& Aldroubi, 1996). 
Early diagnosis of acute Myocardial Infarction (MI) is of vital importance for patients attending the emergency department with chest pain, as there are large benefits for immediate treatment of acute MI patients. With appropriate therapy, the size of an infarct can be reduced which helps in preserving long-term cardiac function. On the contrary, without proper treatment the result may be severe cardiac damage that significantly reduces the prognosis for the patient. Computer-based ECG interpretations for the detection of acute MI are, therefore, of importance as they can improve the early diagnosis of acute MI (Haraldsson et al., 2004).

\section{ECG Data Collection and Sample Selection}

The ECG data for the present work were collected from Al-Kadhimya Teaching Hospital and from Al-Iskandaryia General Hospital in Baghdad. The ECG trace papers were recorded on an A4 trace paper containing the 12 ECG leads. The recorded ECGs were taken from MAC 1200 device. The collected ECG traces contain many diseases like MI and ischaemia (anterior, lateral and inferior wall infarction), ventricular hypertrophy and heart block. Sixty seven ECG traces had been recorded from these two hospitals. Some of them are corrupted by base line wander noise. All the ECG records had been diagnosed with the help of expert cardiologist from Al-Iskandaryia General Hospital. The job of the cardiologist was to diagnose the sixty seven ECG traces and to assess whether they are normal or abnormal.

In the present work, since most of the collected cases contain normal and inferior infarction, the inferior MI and ischaemia were selected to be analyzed by our proposed classification system. Forty-three normal and abnormal subjects (patients with inferior MI) had been selected from the sixty seven cases. These records were displayed on ECG graph paper to be analyzed in the present work. The next step was the conversion of the ECG paper into image file in the computer by using high-resolution scanner. The resulting image file was saved as a bitmap image. The horizontal and vertical resolutions were both set to 150 dots per inch (dpi). The resulting image size is $1230^{*} 1630$ pixels. This procedure was done for all the collected ECG papers.

\section{Methodology}

The block diagram of the proposed system is shown in Fig.1. The following sub-sections will describe each component of the block diagram in detail.

\subsection{Lead Selection}

A single lead ECG is selected from the total ECG image. Lead III has been selected to be processed for the next operations because the inferior infarction appears only in lead III and aVF lead. The selected lead has a length of 13 large squares of the ECG image. The extracted lead III picture is shown in Fig. 2. 


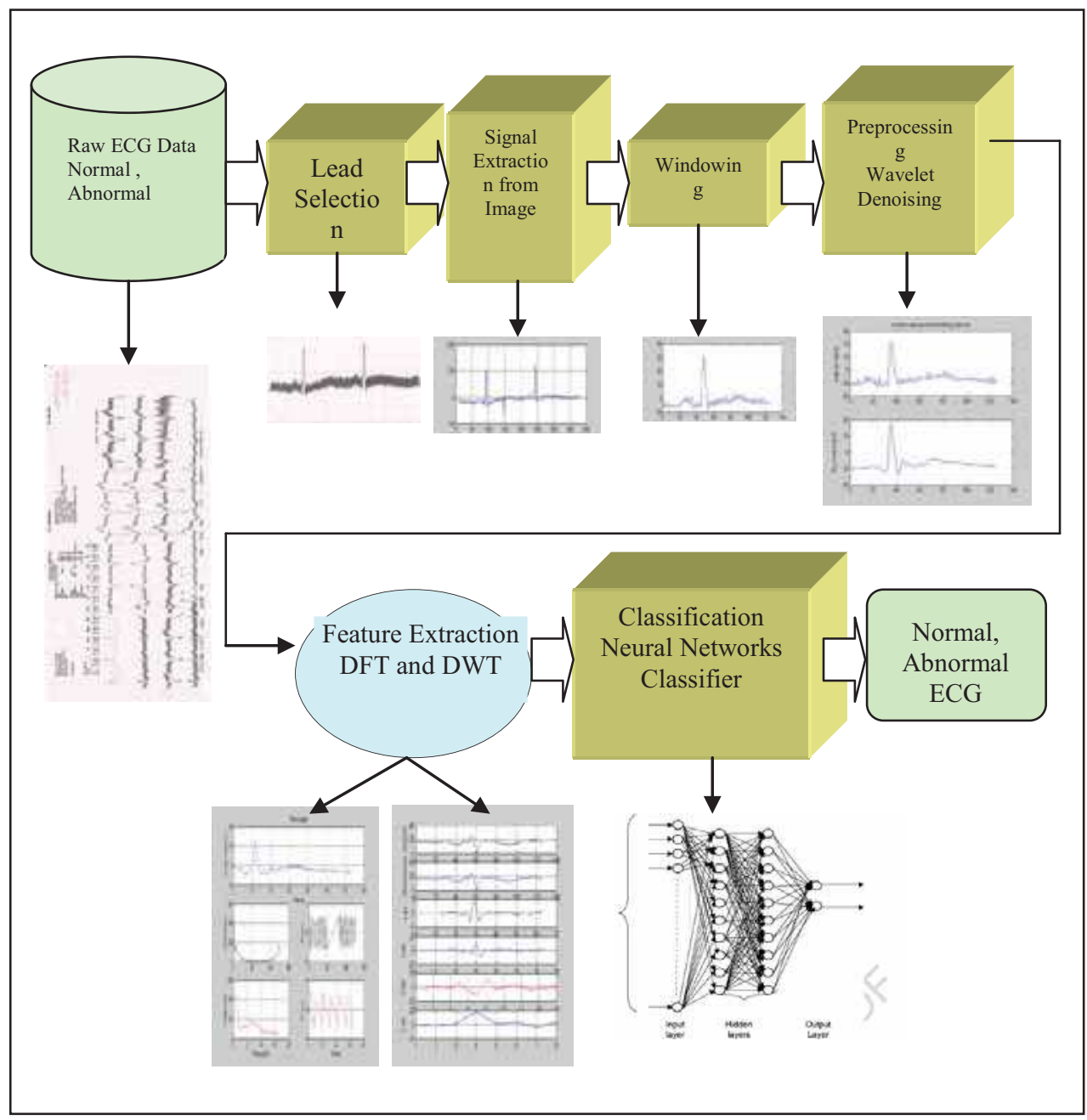

Fig. 1. Block diagram of the ECG classification system

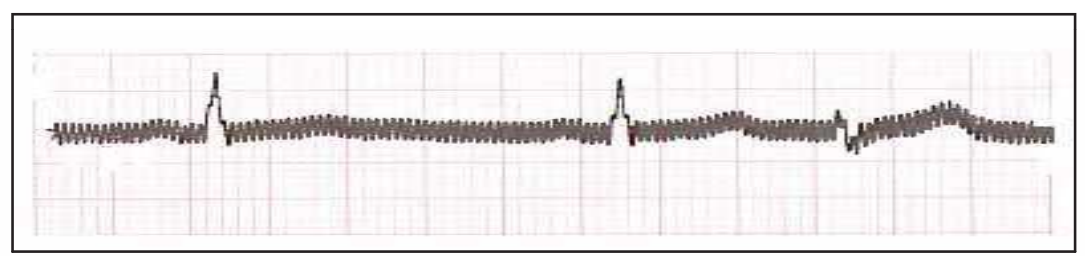

Fig. 2. Lead III picture selected from the total ECG image of sample No. 7 


\subsection{Signal Extraction from Image and Heart Rate Calculation}

The multi-beat lead image in the previous block is converted into a multi- beat signal by the signal extraction block. The flowchart of signal extraction from the image is shown in Fig. 3. The multi-gray leveled ECG image is separated into a single gray level image. Then, scanning for the gray levels between 0 and 200 is done to capture the signal and exclude the background. The gray levels above 200 are discarded based on histogram technique to remove the background. The resulted lead III multi-beat signal is shown in Fig. 4. From this signal, the heart rate is calculated by measurement of R-R interval in large squares since it has a multi-beat by using the formula (Guyton \& Hall, 2000).

$$
H R=300 / R-R \text { (distance in large squares) }
$$

The heart rate is measured for all ECG data and it is expressed in bmp. After heart rate calculation, the algorithm can decide whether the subjects had a tachycardia, bradycardia or normal heart rate.

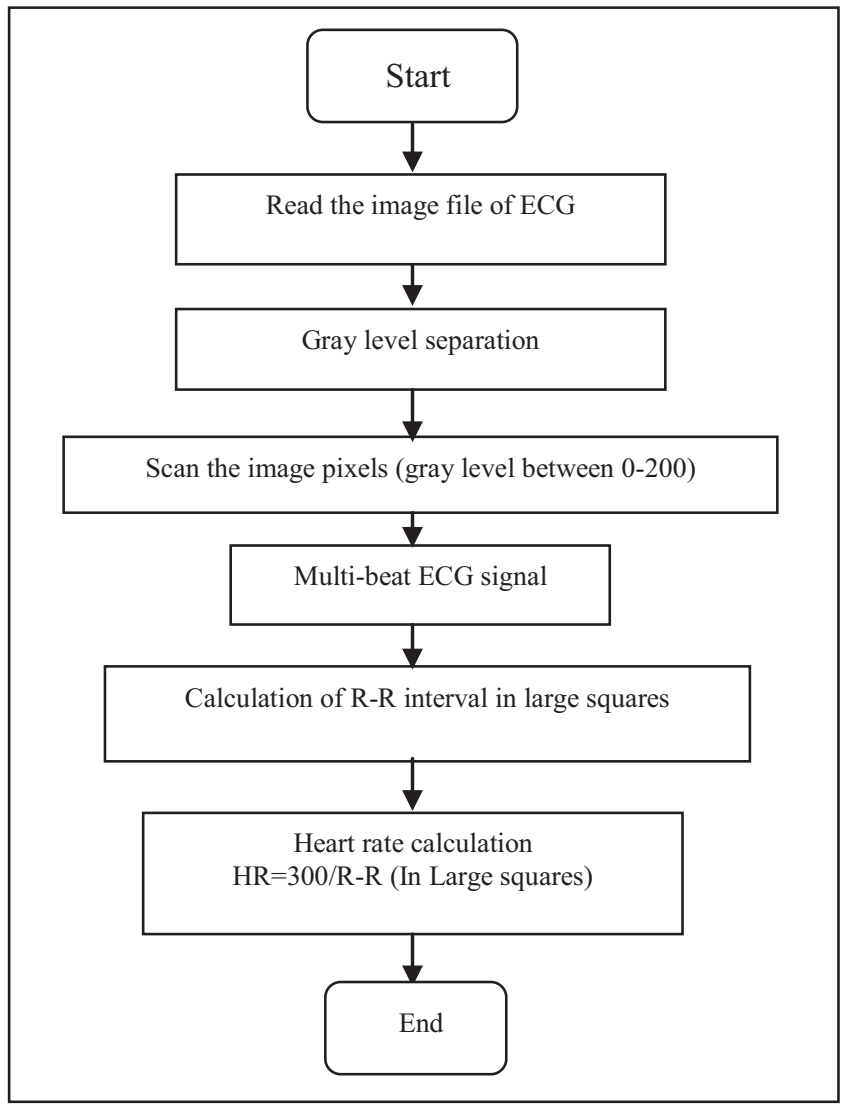

Fig. 3. Flowchart of signal extraction from image and heart rate calculation 


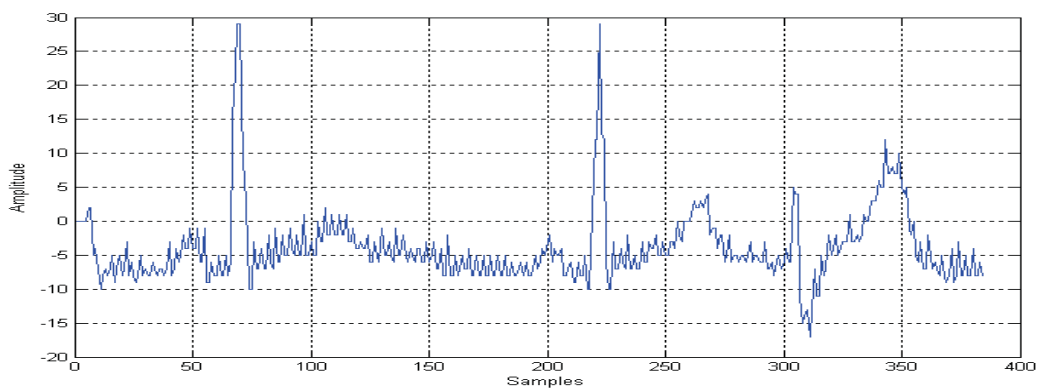

Fig. 4. Extracted lead III multi-beat signal from lead III image of sample No. 7

\subsection{Windowing}

As the shape of each beat in ECG waves is asymmetric, P-QRS-T complexes are selected by using windows with the range 80 samples before the R-wave maximum point to 48 samples after the R-wave maximum. This is to extract a single beat ECG signal from the multi-beat data. The resulting single beat ECG is shown in Fig. 5 . The signal has been normalized to 1 as shown in Fig. 6.

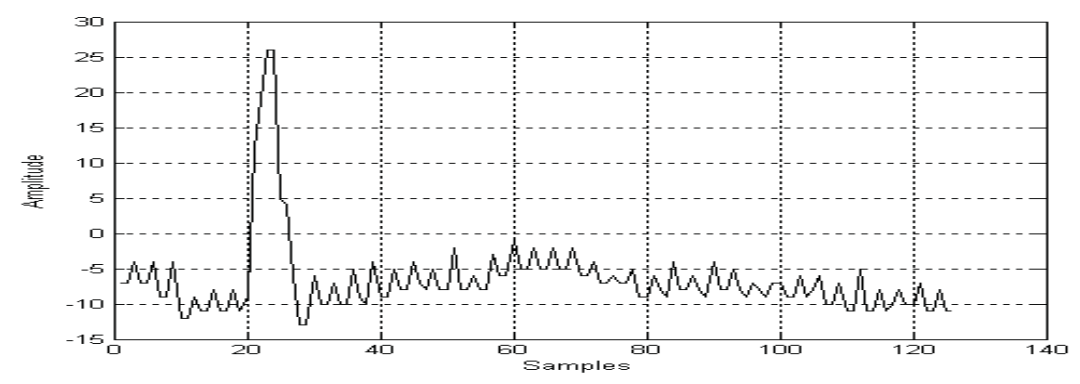

Fig. 5. Single beat lead III signal of sample No. 7

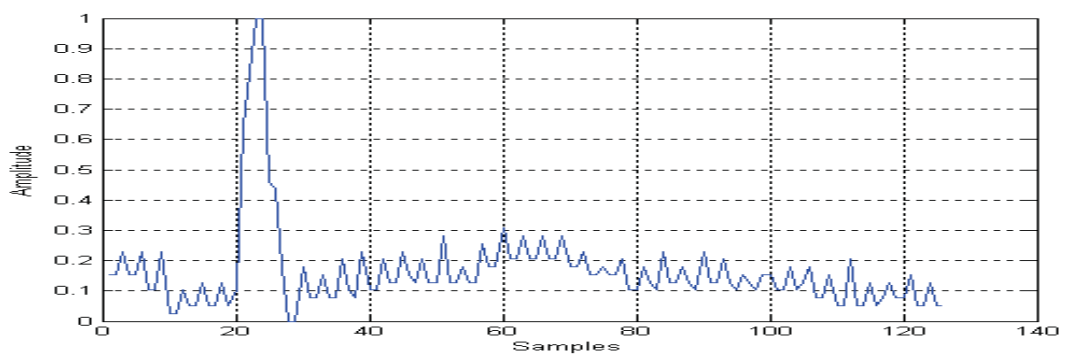

Fig. 6. Normalized single beat signal of sample No. 7 


\subsection{Wavelet Denoising}

Wavelet denoising (WD) method or nonlinear wavelet filtering is based on taking Discrete Wavelet Transform (DWT) of a signal, passing the transform through a threshold, which removes the coefficients below a certain value, then taking the inverse DWT, as illustrated in Fig. 7. The method is able to remove noise and achieve high compression ratios because of the "concentrating" ability of the wavelet transform. The DWT localizes the most important spatial and frequency features of a regular signal in a limited number of wavelet coefficients (Novák, 2000).

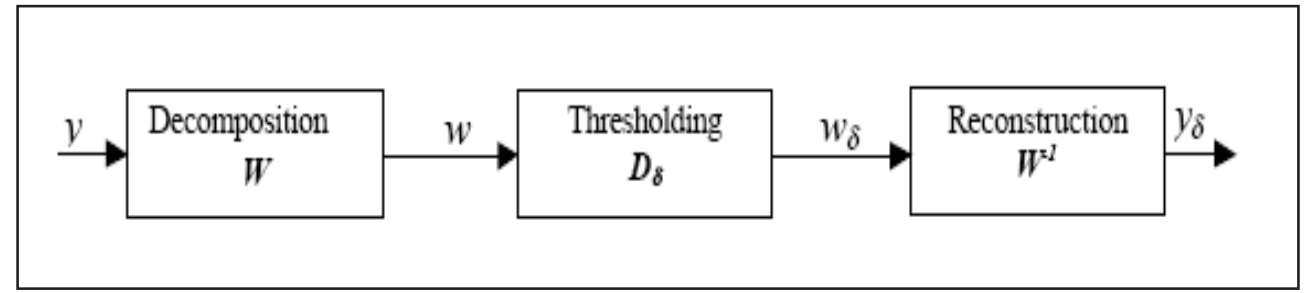

Fig. 7. Basic denoising concept

The general wavelet denoising procedure is as follows (Graps, 1995):

1. Apply wavelet transform to the noisy signal to produce the noisy wavelet coefficients to the desired level.

2. Select appropriate threshold limit at each level and threshold method to best remove the noise.

3. Take the inverse wavelet transform of the threshold wavelet coefficients to obtain a denoised signal.

\subsubsection{Threshold method}

The nonnegative garrote shrinkage function which was first introduced by Breiman (Brieman, 1995); (Poornachandra \& Kumaravel, 2008) is defined as:

$$
\delta_{\lambda}^{G}(x)=x\left\{-(\lambda / x)^{2}\right\}=\left\{\begin{array}{cll}
0 & \text { for } & |x| \leq \lambda \\
x-\lambda^{2} / x & \text { for } & |x|>\lambda
\end{array}\right.
$$

Where $\delta_{\lambda}{ }^{G}(x)$ is the non-negative garrote shrinkage function. A garrote shrinkage function is plotted in Fig. 8. The shrinkage function $\delta_{\lambda}{ }^{G}(x)$ is continuous (like the soft shrinkage, therefore more stable than hard), and approaches the identity line as $|x|$ gets large (close to the hard shrinkage, smaller bias than the soft shrinkage for large coefficient). The nonnegative garrote shrinkage function provides a good compromise between the hard and the soft shrinkage functions. 


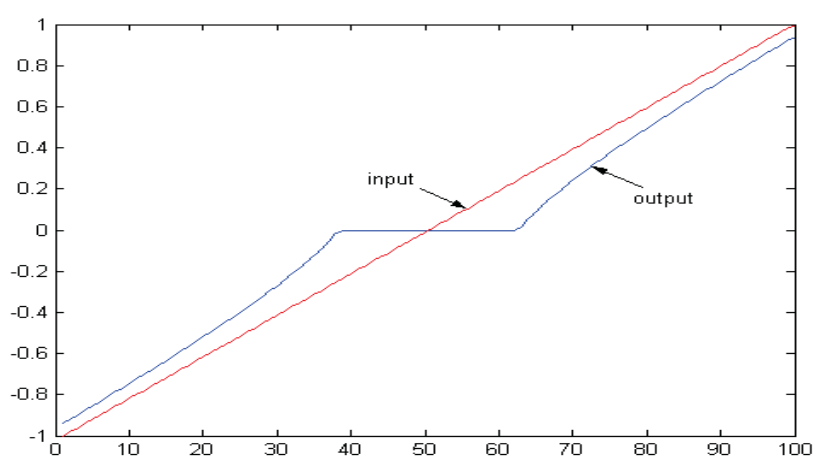

Fig. 8. Garrote thresholding function

\subsubsection{Threshold Selection Rules}

There are three main threshold selection rules (Graps, 1995).

a) Rigsure: threshold is selected using the principle of Stein's Unbiased Risk Estimate (SURE) quadrature loss function. An estimate of the risk can be obtained for a particular threshold value $\lambda$. Minimizing the risks in $\lambda$ gives a selection of the threshold value.

b) Universal: Fixed form threshold yielding minimax performance multiplied by a small factor proportional to the length of the signal.

c) Heursure: Threshold is selected using a mixture of the first two methods. In the present work, Heursure is used as a threshold selection rule.

The original noisy ECG signal and the new denoised signal are shown in Fig. 9.

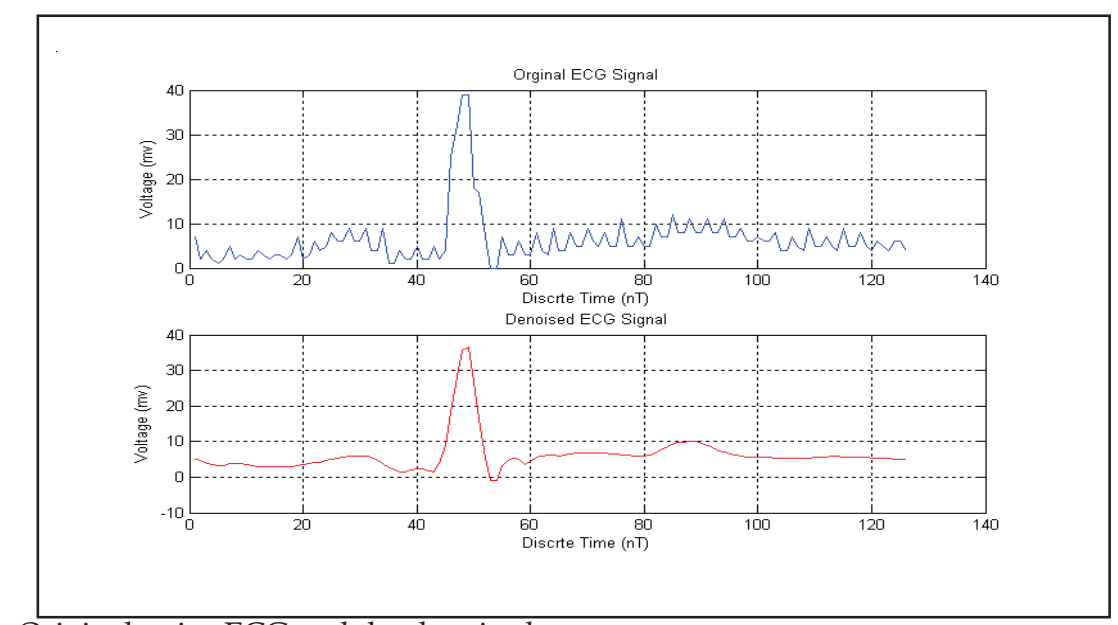

Fig. 9. Original noisy ECG and the denoised one 


\subsection{Feature Extraction}

\subsubsection{Feature Selection}

It is basically impossible to apply any classification method directly to the ECG samples, because of the large amount and the high dimension of the examples necessary to describe such a big variety of clinical situations. A set of algorithms from signal conditioning to measurements of average wave amplitudes, durations, and areas, is usually adopted to perform a quantitative description of the signal and a parameter extraction. On this set of extracted ECG parameters, several techniques for medical diagnostic classification are then applied, such as probabilistic approaches, heuristic models, and knowledge-based systems. The aim of this work was to determine suitable input feature vectors which would discriminate between normal and abnormal MI beats (Al-Naima et al., 2008).

\subsubsection{Reasons for the Use of Wavelet}

This is the most interesting question for most of the users. The wavelet has one or two parameters. Because wavelets have so many constraints that are not associated with the signal, but more with mathematical and calculation limitations, it is virtually impossible to blindly select a wavelet. Wavelets are usually chosen on the basis of "If you see what you need to see, then that's that, if not, then try something else". The most general-purpose usable wavelet is Daubechies (Belgacem et al., 2003).

\subsubsection{DWT Coefficients Extraction}

In the present work $\mathrm{Db} 4$ and Haar wavelet have been used as the mother wavelets. MATLAB software package version 7 used to extract the DWT coefficients. For achieving good time-frequency localization, the preprocessed ECG signal is decomposed by using the DWT up to the fourth level. The smoothing feature of Haar wavelets and Db4 made them more suitable to ECG changes and the feature set is composed of level 1,2,3, 4 coefficients $\mathrm{cd} 1, \mathrm{~cd} 2 \mathrm{~cd} 3, \mathrm{~cd} 4$ and ca4. Most of the energy of the ECG signal lies between $0.5 \mathrm{~Hz}$ and 40 $\mathrm{Hz}$. This energy of the wavelet coefficients is concentrated in the lower sub-bands ca4, cd4, and $\mathrm{cd} 3$. The level 1, 2 coefficients $\mathrm{cd} 1$ and $\mathrm{cd} 2$ are the most detail information of the signal and they are discarded since the frequency band covered by these levels contains much noise and is less necessary for representing the approximate shape of ECG. The frequencies covered by these levels were higher than frequency content of the ECG. Coefficients $\mathrm{cd} 3$ and cd4 represent the highest frequency components and ca4 represent the lowest one.

For the Db4 wavelets, cd3 and cd4 having lengths of 21 and 14 coefficients are generated respectively. The DWT coefficients of Db4 wavelets of ECG segment of sample no. 7 are shown in Fig. 10. For the Haar wavelets, $\mathrm{cd} 3$ and cd4 having lengths of 16 and 8 coefficients are generated respectively. The obtained feature vectors form $\mathrm{Db} 4$ and Haar wavelets decomposition are used as an input to the NN classifier. The above procedure of decomposition is done for the 43 ECG segments for both normal and inferior MI patients. 


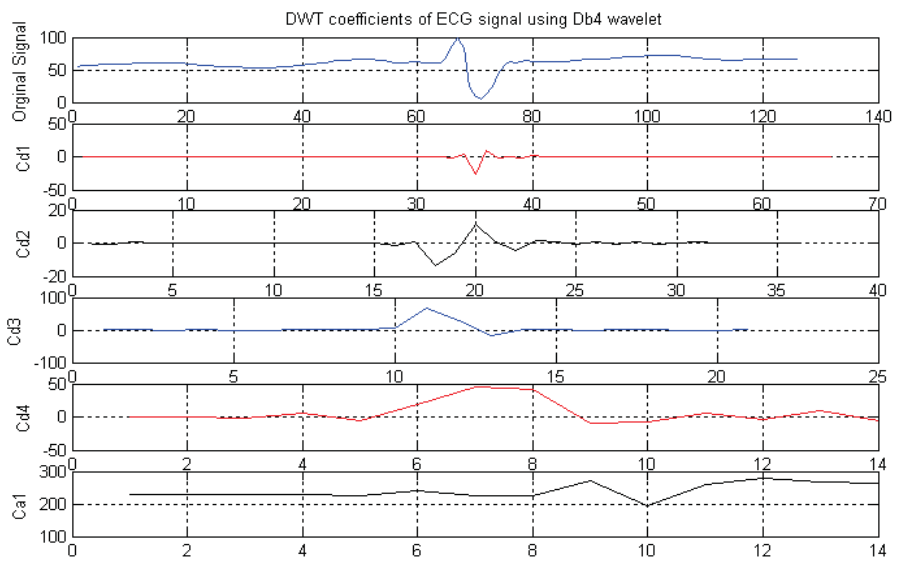

Fig. 10. The DWT coefficients of ECG Db4 wavelet of level-4 of sample No. 7

\subsubsection{Discrete Fourier Transform}

Discrete Fourier Transform of each data set was performed after getting the sampling period to observe both frequency and phase response properties of every ECG signals. Each ECG segment is analyzed by DFT of 128 points. Due to symmetry of the DFT, only 64 points are considered. Thus, the Fourier magnitude and phase of 64 points length have been obtained. To reduce the dimensionality, 16 points are selected from the 64 points sample of the Fourier magnitude. For the phase, a set of 32 points are selected from the 128 points phase. Both the 16 samples Fourier magnitude and 32 samples phase are used as feature vectors to be introduced to the NN classifier. The above procedure is done for each noisy and denoised ECG segments of the 43 cases of normal and patients with MI. The Fourier magnitude and phase for denoised ECG segment of sample no. 7 are shown in Fig. 11.

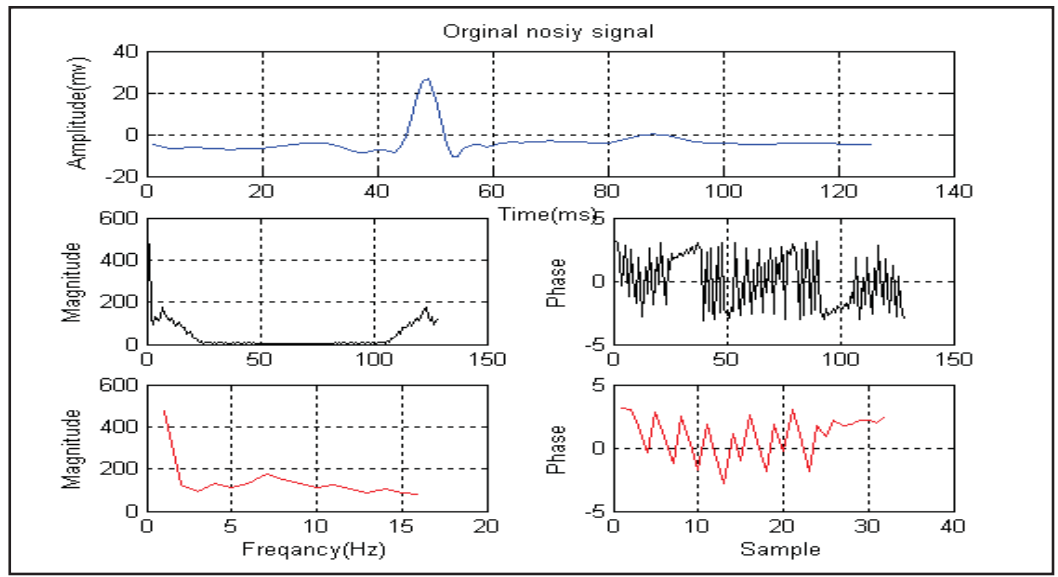

Fig. 11. The Fourier magnitude and phase for the denoised ECG signal 


\subsection{Neural Networks Classifier}

In the present work, the neural networks are used for the classification purposes. The neural networks derive their power due to their massively parallel structure, and an ability to learn from experience. They can be used for fairly accurate classification of input data into categories, provided they are previously trained to do so. The accuracy of the classification depends on the efficiency of training. The knowledge gained by the learning experience is stored in the form of connection weights, which are used to make decisions on fresh input. Three issues need to be settled in designing an ANN for a specific application:

1. Topology of the network.

2. Training algorithm.

3. Neuron activation functions.

In the topology we adopted, the number of neurons in the input layer was fixed by the number of elements in the input feature vector. Therefore the input layer had 16 neurons for both the first and the third ANN classifiers, 21 neurons for the second, and 32 neurons for the fourth one using DWT (Db4), DWT (Haar), Fourier magnitude, Fourier phase respectively. The output layer was determined by the number of classes desired. In our study, the unique neuron of the output layer corresponds to the normal and MI beats.

The proposed network was trained with all 45 cases (26 normal and 19 abnormal cases). These 45 cases are fed to the four feed forward neural network proposed in this study.

MATLAB software package version 7 is used to implement the software in the current work. When the training process is completed for the training data (45 cases), the last weights of the network were saved to be ready for the testing procedure. Learning rate is set to 0.5 , the output of the network was (-1) for the class normal and (1) for the class abnormal. The training algorithm used for this network is the Back Propagation Algorithm (BPA). The performance goal was met at 2300 epochs after a training time of $45 \mathrm{sec}$. The summary of the back-propagation algorithm applied in the present work can be described as:

1. Initialization: Assuming no prior information is available, the synaptic weights and thresholds have picked to a random value.

2. Presentations of the training examples The network is presented with an epoch of training examples. For each example in the set, ordered in some fashion, the sequence of forward and backward computations described under points 3 and 4 is performed.

3. Forward computation

4. Backward computation

5. Iteration The forward and backward computations under points 3 and 4 are iterated by presenting new epochs of training examples to the network to reach the stopping criteria.

The testing process is done for 20 cases (12 normal and 8 abnormal). These 79 cases are fed to the proposed network and the output is recorded for calculation of the sensitivity, specificity and accuracy of prediction. 
The accuracy of the classification depends on the efficiency of training. The knowledge gained by the learning experience is stored in the form of connection weights which are used to make decisions on fresh input.

Classification of MI is a complicated problem. To solve this two hidden layers are taken in a feed forward neural network. The single hidden layer is set for our four neural classifiers as follows: For the DWT (Db4) NN and Fourier magnitude NN, the hidden layer consists of four neurons. For the DWT (Haar) NN, the hidden layer consists of six neurons. And for the Fourier phase NN, the hidden layer consists of 8 neurons. The BPA is a supervised learning algorithm, which aims at reducing the overall system error to a minimum. The connection weights are randomly assigned at the beginning and progressively modified to reduce the overall mean square system error. The weight updating starts with the output layer, and progresses backwards. The weight update aims at maximizing the rate of error reduction, and hence, it is termed as 'gradient descent' algorithm. It is desirable that the training data set be large in size, and also uniformly spread throughout the class domains. In the absence of a large training data set, the available data may be used iteratively, until the error function is reduced to an optimum level. For quick and effective training, data are fed from all classes in a routine sequence, so that the right message about the class boundaries is communicated to the ANN.

Before the training process is started, all the weights are initialized to small random numbers. This ensured that the classifier network was not saturated by large values of the weights. In this experiment, the training set was formed by choosing 15 normal beats and 12 MI beats obtained from the selected cases.

The sigmoid function was used as the neural transfer function. The most important reason for choosing the sigmoid as an activation function for our networks is that the sigmoid function $f(x)$ is differentiable for all values of $x$, which allows the use of the powerful BPA.

\section{Results and Discussion}

The performance of the algorithm was tested by computing the percentages of the three parameters; Sensitivity (SE), Specificity (SP) and Accuracy (AC) as follows (Al-Timemy, 2008; Al-Timemy \& Al-Namia, 2009):

$$
\begin{gathered}
S E=\frac{T P}{(T P+F N)} \times 100 \\
S P=\frac{T N}{(T N+F P)} \times 100 \\
A C=\frac{(T P+T N)}{(T N+T P+F N+F P)} \times 100
\end{gathered}
$$


Where TP is the number of true positives, TN is the number of true negatives, $\mathrm{FN}$ is the number of false negatives, and FP is the number of false positives. The true positives (TP), false positives $(\mathrm{FP})$, true negatives $(\mathrm{TN})$, and false negatives $(\mathrm{FN})$ are defined appropriately as shown below:

FP: Classifies normal as abnormal.

TP: Classifies abnormal as abnormal.

FN: Classifies abnormal as normal.

TN: Classifies normal as normal.

In our study, the unique neuron of the output layer corresponds to the normal and infarction beats. In practice, the number of neurons in the hidden layer varies according to the specific recognition task and is determined by the complexity and amount of training data available. If too many neurons are used in the hidden layer, the network will tend to memorize the data instead of discovering the features. This will result in failing to classify new input data. Using a trial-and-error method, we tested hidden layers varying between two and 20 neurons. The optimum number of neurons in the hidden layer was found to be five for the first ANN classifier, three for the second and two for the last one. The resulted accuracy, sensitivity and specificity for DFT-NN and DWT-NN are shown in Table 1.

\begin{tabular}{|c|c|c|c|c|}
\hline Disease & cases & Accuracy & Sensitivity & Specificity \\
\hline DFT-NN & 20 & $85 \%$ & $80 \%$ & $90 \%$ \\
\hline DWT-NN & 20 & $95 \%$ & $90 \%$ & $90 \%$ \\
\hline
\end{tabular}

Table 1 . The results after training of the proposed network

\section{Conclusion}

ECG signals of the human generated by the conduction system of the heart are usually nonstationary signals. A method based on image processing techniques was presented for data acquisition of the ECG cases. This method of obtaining ECG samples was shown to be efficient in ECG samples acquisition. In the present work, classification of ECG patterns was achieved by means of DWT and DFT combined with BP NN. In its current form, BPA uses the gradient descent to train the network.

The objective is to minimize the BP error to reach the desired response. Denoising process was adopted to remove different types of noise corrupting the ECG samples. The ECG signal can be used as a reliable indicator of heart diseases. In the present work, the DWT, DFT and the NN classifier are presented as diagnostic tools to aid the physician in the analysis of heart diseases. A wavelet based NN classifier has been proposed for MI classification. The feature set has been carefully chosen to have enough information for good accuracy. This feature set is a subset of DWT coefficients based on 'Db4' and 'Haar' wavelets. 


\section{References}

Acharya, R., Kumar, A., Bhat, P. S., Lim, C. M., Iyengar, S. S., Kannathal, N. \& Krishnan, A. S. M. (2004). Classification of Cardiac Abnormalities using Heart Rate Signal. Medical \& Biological Engineering \& Computing, 42

Afsar, F. A. \& Arif, M. (2008). Robust Electrocardiogram Beat Classification using Discrete Wavelet Transform. Bioinformatics and Biomedical Engineering, 2008. ICBBE 2008. The 2nd International Conference on.

Al-Naima, F. M. M, Al-Timemy, A. H. A. \& Mahdi, S. S. (2008). Data Acquisition for Myocardial Infarction Classification based on Wavelets and Neural Networks. 5th International Multi-Conference on Systems, Signals and Devices, 2008. IEEE SSD 2008. 20-22 July 2008, pp. 1-6, Amman, Jordan, ISBN:978-1-4244-2205-0

Al-Timemy, A. H. A. (2008). Self-Organization Maps for Prediction of Kidney Dysfunction. Proc. 16th Telecommunications Forum, TELFOR, Belgrade, Serbia, 2008, pp. 775-778

Al-Timemy, A. H. A. \& Al-Naima, F. M. M. (2009). Comparison of Different Neural Network Approaches for the Prediction of Kidney Dysfunction. International Journal of Biological and Medical Sciences Vol. 5, No. 1, pp. 15-20, ISSN: 2070-3791

Anan, K., Dowla, F. \& Rodrigue, G. (1995). Vector Quantization of ECG Wavelet Coefficients. Signal Processing Letters, IEEE, 2, pp.129-131

Belgacem, N., Chikh, M. A., \& Bereksi-Rreguig, A. (2003). Detection of Cardiac Arrhythmias By Neural Networks, The 5th International Conference on Enterprise Information Systems, ICEIS 2003, Angers, France, April 22-26, 2003, Angers, France

Breiman, L. (1995). Better Subset Regression Using the Nonnegative Garrote. Technometrics, Vol. 37, No. 4, Nov., 1995, pp. 373-384

Graps, A. (1995). An Introduction to Wavelets. IEEE Computational Science and Engineering Vol. 2, No. 2 (1995), pp. 1-19

Güler, I. \& Übeyli, E. D. (2004). Application of Adaptive Neuro-fuzzy Inference System for Detection of Electrocardiographic Changes in Patients with Partial Epilepsy using Feature Extraction. Expert Systems with Applications, Vol. 27, No. 3, (2004), pp. 323330

Guyton, A. \& Hall, A. J. E. (2000). Text Book of Medical Physiology, W.B. Saunders Company, U.S.A

Haraldsson, H.; Edenbrandt, L. \& Ohlsson, M. (2004). Detecting Acute Myocardial Infarction in the 12-lead ECG using Hermite Expansions and Neural Networks. Artificial Intelligence in Medicine, Vol. 32, No. 2 (2004), pp. 127-136

Karuiannis, N. B. \& Venetsanepouious, A. N. (1997). Artificial Neural Network, Learning Algorithm, Performance, Evolution and Applications, Kluwer Academic Publisher, London

Kim, M. J., Han, J. S.; Park, K. H., Bang, W. C. \& Bien, A. Z. Z. (2001). Classification of Arrhythmia Based on Discrete Wavelet Transform and Rough Set Theory. International Conference on Control Automation and Systems, (ICCAS 2001), Oct 17-21, 2001, Jeju National University, Jeju, Korea

Novak, D. (2000). ECG Processing using Wavelets, Final Year Thesis, Valencia Technical University and Czech Technical University in Prague, Czech Republic

Owis, M. I.; Abou-Zied, A. H.; Youssef, A. B. M. \& Kadah, Y. M. (2002). Study of Features based on Nonlinear Dynamical Modeling in ECG Arrhythmia Detection and 
Classification. IEEE Transactions on Biomedical Engineering, Vol. 49, No.7, (2002), pp. 733-736

Poornachandra \& S., Kumaravel, N. (2008). A Novel Method for the Elimination of Power Line Frequency in ECG Signal using Hyper Shrinkage Function. Digital Signal Processing, Elsevier,. Vol. 18,(2008), pp. 116-126

Prasad, G. K. \& Sahambi, J. S. (2003). Classification of ECG Arrhythmias using Multiresolution Analysis and Neural Networks. Proc. IEEE Conf. on Convergent. Technologies (Tecon2003), Bangalore, India, Vol. 1, pp. 227-231

Unser, M. \& Aldroubi, A. (1996). A Review of Wavelets in Biomedical Applications. Proceedings of the IEEE, Vol. 84, No. 4, pp. 626-638 


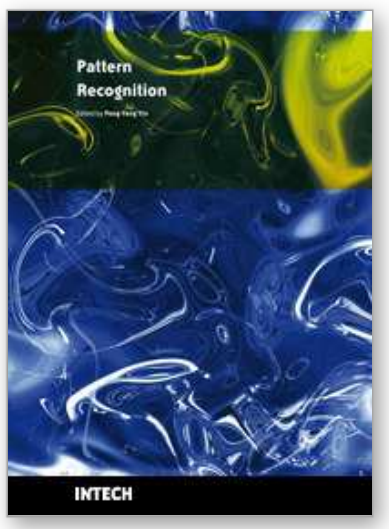

\section{Pattern Recognition}

Edited by Peng-Yeng Yin

ISBN 978-953-307-014-8

Hard cover, 568 pages

Publisher InTech

Published online 01, October, 2009

Published in print edition October, 2009

For more than 40 years, pattern recognition approaches are continuingly improving and have been used in an increasing number of areas with great success. This book discloses recent advances and new ideas in approaches and applications for pattern recognition. The 30 chapters selected in this book cover the major topics in pattern recognition. These chapters propose state-of-the-art approaches and cutting-edge research results. I could not thank enough to the contributions of the authors. This book would not have been possible without their support.

\section{How to reference}

In order to correctly reference this scholarly work, feel free to copy and paste the following:

Fawzi Al-Naima and Ali Al-Timemy (2009). Neural Network Based Classification of Myocardial Infarction: A Comparative Study of Wavelet and Fourier Transforms, Pattern Recognition, Peng-Yeng Yin (Ed.), ISBN: 978953-307-014-8, InTech, Available from: http://www.intechopen.com/books/pattern-recognition/neural-networkbased-classification-of-myocardial-infarction-a-comparative-study-of-wavelet-and-four

\section{INTECH}

open science | open minds

\section{InTech Europe}

University Campus STeP Ri

Slavka Krautzeka 83/A

51000 Rijeka, Croatia

Phone: +385 (51) 770447

Fax: +385 (51) 686166

www.intechopen.com

\section{InTech China}

Unit 405, Office Block, Hotel Equatorial Shanghai

No.65, Yan An Road (West), Shanghai, 200040, China

中国上海市延安西路65号上海国际贵都大饭店办公楼405单元

Phone: +86-21-62489820

Fax: $+86-21-62489821$ 
(C) 2009 The Author(s). Licensee IntechOpen. This chapter is distributed under the terms of the Creative Commons Attribution-NonCommercial-ShareAlike-3.0 License, which permits use, distribution and reproduction for non-commercial purposes, provided the original is properly cited and derivative works building on this content are distributed under the same license. 